\title{
INTEGRATED SYSTEM INTEROPERABILITY TESTING WITH APPLICATIONS TO VOIP
}

\author{
Nancy Griffeth, Ruibing Hao, David Lee*, Rakesh K. Sinha \\ Bell Laboratories \\ Murray Hill, New Jersey \\ * Bell Laboratories Research China \\ Beijing, China \\ \{nancyg,rbhao,leedavid,rks1\}@@lucent.com
}

\begin{abstract}
This work has been motivated by the need to test interoperability of systems carrying voice calls over the IP network. Voice over IP (VoIP) systems must be integrated and interoperate with the existing Public Switched Telephone Network (PSTN) before they will be widely adopted. Standards have been developed to address this problem, but unfortunately different standards bodies and commercial consortiums have defined different standards. Furthermore, the prevailing commercial standard (H.323) is incomplete, complex, and presents the implementers with "vendors latitudes". As a result, there is no guarantee that the integrated VoIP systems would interoperate properly even if the implementations are all H.323-compliant. Interoperability testing has become indispensable.

We discuss criteria that cover all the required patterns of "interoperating behavior." We want to test all such system interoperations. On the other hand, test execution in real environment is expensive, and we want to minimize the number of tests while maintaining the coverage.

We present a general method for automatic generation of test cases, which cover all the required system interoperations and contain a minimal number of tests. We study data structures and efficient test generation algorithms, which take time proportional to the total test case size. We report experimental results on VoIP systems.
\end{abstract}

Keywords: VoIP, H.323 protocol, integrated system, interoperability testing, coverage, redundancy

The original version of this chapter was revised: The copyright line was incorrect. This has been corrected. The Erratum to this chapter is available at DOI: 10.1007/978-0-387-35533-7_26

Tommaso Bolognesi and Diego Latella (Eds.), Formal Methods for Distributed System Develcpment. (c) 2000 IFIP International Federation for Information Processing.

Published by Kluwer Academic Publishers. All rights reserved. 


\section{INTRODUCTION}

With the rapid growth of Internet, more and more new protocols are proposed and new products are developed, such as Voice over IP(VoIP) and Virtual Private Network(VPN). When two or more entities in separate communicating systems are integrated and need to interact with each other to perform a certain task the capability to operate as desired is called interoperability, which is considered an essential aspect of correctness of integrated systems. However, products from different vendors or even from the same vendor often do not interoperate properly. Two main causes of non-interoperation are ambiguity of protocol specification and a vendor's proprietary extensions. Interoperability testing is done to check the interoperations among integrated system implementations.

The research work on interoperability testing can be roughly classified into two categories: general concepts and experiences of interoperability testing, and systematic generation of interoperability test suites [7].

Most of the early work $[6,4,14]$ (beginning of 90s) belongs to the first category. Majority of the recent research work is related to interoperability test suite derivation. One approach for interoperability test generation is to apply conformance test generation techniques on composed finite state machines, which are constructed from several components systems via a reachability analysis. A finite set of test cases is then selected to test the interoperability. Rafiq et al. [13] was one of the first papers in this field. It proposes the above test generation procedure and also an interoperability test architecture. Kang and Kim [9] proposed a technique for symmetric protocol control portion interoperability testing. However, for practical systems, this system composition approach often leads to state explosion $[12,11,5,8]$. Furthermore, we may not have complete information on all the interoperating systems. For instance, in VoIP systems, we can model end user behavior and $\mathrm{H} .323$ protocols, but we do not have a specification of the communication system, which includes the IP network and is too complex to model.

Our work has been motivated by the need to test interoperability of systems carrying voice calls over the IP network. Voice over IP (VoIP) systems must be integrated and interoperate with the existing Public Switched Telephone Network (PSTN) before they will be widely adopted. We model the system behaviors by extended finite state machines (EFSM), and propose a testing architecture for interoperability testing. Based on the experiences of domain experts, we discuss various criteria that cover the required patterns of interoperations. A key idea in developing the coverage criteria is that interoperability errors are introduced only when the integrated systems are "interacting" with 
each other. Based on this observation, our test generation only requires information about part of the integrated communication systems; it is impossible to obtain, model and analyze the whole systems in practice. For instance, for VoIP interoperability testing, we only need information about end user required behaviors and H.323 interfaces. Consequently, it does not require the modeling and construction of the composition of all the integrated systems, including the IP network, and avoids the state explosion problem while checking all the required interoperations.

We design efficient data structures and test generation algorithms, which guarantee desired coverage and contain a minimal number of tests.

These algorithm are part of a software tool, ITIS, developed at Bell Laboratories. With this tool, we have generated interoperability test cases for: End users vs. the rest of the communication system; and End Users and H.323 vs. the rest of the communication system. For clarity, we only report the experimental results of interoperability testing of the end users behaviors vs the rest of the systems.

The remainder of this paper is organized as follows. After introducing a formal model, we present the interoperability test generation architecture and techniques in Section 2. Section 3 contains more details of the test generation algorithms, which incorporate a range of coverage and redundancy criteria. Experimental results on VoIP are reported in Section 4.

\section{Formal Model}

Communicating system behaviors can often be modeled by extended finite state machines, which are finite state machines extended with variables. We denote a finite set of variables by a vector: $\vec{x}=\left(x_{1}, \ldots, x_{k}\right)$. A predicate on variable values $P(\vec{x})$ returns FALSE or TRUE; Given a function $A(\vec{x})$, an action is an assignment: $\vec{x}:=A(\vec{x})$. Each transition $t$ is a 6-tuple: $t=\left(s_{t}, q_{t}, a_{t}, o_{t}, P_{t}, A_{t}\right)$, where $s_{t}, q_{t}, a_{t}$, and $o_{t}, P_{t}, A_{t}$ are the start state, end state, input, output, predicate, and action respectively. In state $s_{t}$, upon input $a_{t}$, if $\vec{x}$ is valid for $P_{t}$, i.e., $P_{t}(\vec{x})=$ TRUE, then the machine follows the transition $t$, outputs $o_{t}$, changes the current variable values by action $\vec{x}:=A_{t}(\vec{x})$, and moves to state $q_{t}$.

Each combination of a state and variable values is called a configuration. We can construct a reachability graph of configurations starting from the initial configuration. If each variable has a finite number of values (Boolean variables for instance), then there are a finite number of configurations and the reachability graph is an equivalent (ordinary) FSM with configurations as states [10]. Therefore, an EFSM with finite variable domains is a compact representation of an FSM. 


\section{INTEROPERABILITY TEST GENERATION}

When we do interoperability testing, we are concerned only with those failures that occur when integrated systems are interoperating. For instance, consider Voice over IP systems in Figure 1. Two H.323 end-users want to communicate. They each access the communication system through an H.323 gateway, using H.323 protocol. We may want to test the interoperability between H.323 end users and the rest of the systems, i.e., the H.323 gateways and the communication system.

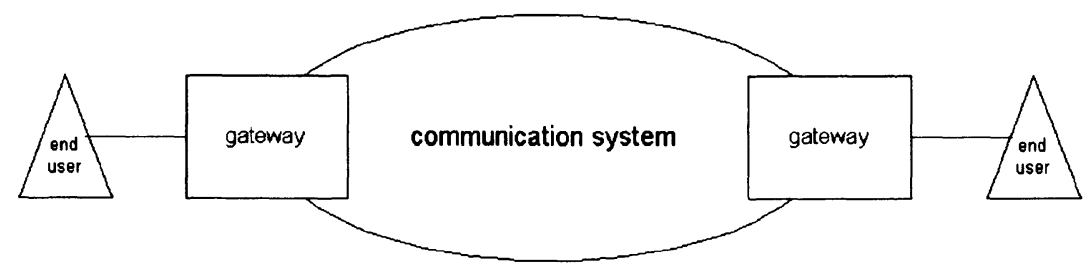

Figure 1 Model of interoperating systems

For interoperability testing, we would like to test all possible system interoperations. For instance, in the case of interoperability of end users and the rest of the system, we might want to test all the interoperations between the end users vs. the rest of the system. For this test, assume that we can access the system through end user behaviors only. We model the joint behaviors of the two end users by an EFSM. Ideally, we want to check every input sequence to the end user EFSM so that all its interoperations with the rest of the system are tested. It is an impossible task. We want to remove the redundant tests (redundancy will be defined in the sequel) and, hopefully, reduce to a small number of tests.

In general, we want to test interoperations of system $A$ and $B$. We have complete information about $A$ but not $B$ or choose not to model $B$, either because the information is not available or is too complex. We can only access the systems through the interface with $A$. Based on our information about $A$, we want to construct a set of non-redundant tests such that all the interoperations between $A$ and $B$ are tested. Our goals are: (1)Completeness: all the interoperations between the two systems are tested; and (2)Non-Redundancy: all the redundant tests are removed to minimize the total number of tests.

Note that interoperability testing is different than conformance testing; even a checking sequence [10] of system $A$ may not be sufficient due to the following reasons. A checking sequence guarantees structural 
isomorphism between the implementation and specification machines under the assumption that the implementation machine has no more states than that of the specification machine. It may not be true for the expected behavior of an integrated system, in which the number of states is unknown. Furthermore, we have no information or only partial information of system $B$. Therefore, for interoperability testing, we need different tests to ensure the coverage of all the interoperations between $A$ and $B$, given only information about $A$.

There are various techniques to remove redundancy. For instance, in the configuration of Figure 1, a key idea in developing the test cases is that interoperability errors will be introduced only when the gateways are actually talking to each other about a call. Thus we can ignore local activities involved in the protocol, such as obtaining information from an end-user. We label as "white" the transitions that can be ignored and label all other transitions as "black." A white transition is purely local, that is, it reads the state of only one gateway and writes that same state. A black transition involves both gateways, because it reads both states, or reads one state and writes the other.

This is illustrated in the call flow for a simple telephone call in Figure 2. The transition Off-hook from Idle state to Dialing state is white, because it involves only the originating gateway and the end user. The Dial transition from Dialing to Calling is black, because the state is changed in both gateways. Similarly, the Off-hook transition which connects the call changes the state in both gateways from Calling to Connected.

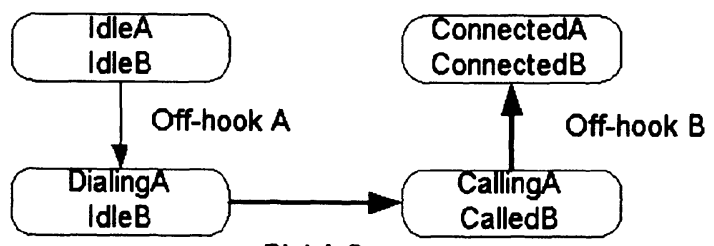

Dial A B

Figure 2 Partial call flow of a telephone call

The test cases that we generate focus only on black edges. The purpose of white edges is to interconnect the sequence of black edges.

\subsection{TEST GENERATION STRATEGY}

We want to test interoperability of system $A$ and $B$, assuming we have complete information about $A$. We can model it by an EFSM and construct its reachability graph, which is a directed graph, a transition diagram [10]. Throughout the rest of the paper, we will be using graph 
terminology. For example, we will denote the initial state as the "source node", transitions as "edges", etc.

Ideally, we want to cover (test) all possible execution sequences, often called scenarios. Therefore, covering all possible execution sequences requires that we cover all the branches and all the possible paths. Consequently, we cover all the interoperations from $A$ to $B$, and this would be our interoperability coverage criteria. However, it is impossible; in general, transition diagrams often contain cycles and, therefore, will have infinitely many distinct paths. We need to remove the redundant paths, if possible, and reduce the number of tests to a manageable size.

Our test generation process contains three steps:

step 1 Generate all possible acyclic paths, i.e. paths without any repeated vertices.

step 2 Generate all possible simple cycles, i.e. cycles that do not contain any smaller cycles.

step 3 "Combine" the paths (from step 1) and simple cycles (from step

2) to generate the final set of paths.

The number of paths and cycles generated in the first two steps is finite. In a later section, we will describe various criteria for removing the redundant cycles and paths. The goal of the "combine" process in step 3 is to generate a finite number of paths that cover all necessary scenarios. This is done as follows:

First we include all the acyclic paths. Then we generate additional cyclic paths as follows. For each acyclic path $P$, find all the simple cycles that share a node with $P$. Let these cycles be $C_{1}, C_{2}, \ldots, C_{k}$ and let $v_{i}, 1 \leq i \leq k$, be a node common to $C_{i}$ and $P$. Then generate a new (cyclic) path by replacing node $v_{i}$ in $P$ with cycle $C_{i}$.

Now we elaborate on steps 1 and 2. We first find all the strongly connected components (SCC) of the transition graph. This has two advantages: we know that any cycle is completely contained inside an SCC. So we can perform step 2 by looking at each SCC in turn and finding all simple cycles within this SCC. The second advantage is that we can "shrink" each SCC into a node and obtain a Directed Acyclic Graph (DAG), i.e., a graph without any cycles [3]. This naturally translates into a two phase algorithm for step 1. First, generate all acyclic paths on the resulting DAG and then replace each SCC on any given path with a set of acyclic paths within the SCC.

\subsection{NEXT-TRANSITION-TREE}

We describe a simple data structure, next-transition-tree, that will be convenient for both steps 1 and 2. The data structure can be defined for any graph but in our application, the graph will always be an SCC. 
For any node $v$, next-transition-tree $(v)$ stores all acyclic paths from $v$ to other vertices in its SCC. The tree has $v$ as its root and its height is equal to the number of nodes in the SCC containing $v$. The children of $v$ are all the nodes in its SCC that have a direct edge from $v$. In general, the children of any node $u$ are all the nodes in its SCC that have a direct edge from node $u$. Please note that a node may appear multiple times in this tree. (Precisely speaking we should say that each node has a label where the labels are not necessarily unique. The root node has label $v$. A node labeled $u$ has as many children as the outdegree of $u$ in its SCC and these children are labeled with the corresponding nodes in the SCC.)

We do not need a next-transition-tree for each node in the graph. We need it only for a few of the nodes; the details are in Section 4 . For ease of presentation, we assume that we maintain a separate next-transitiontree for each node. The actual implementation will have lots of shared pieces among next-transition-trees belonging to the same SCC.

\subsection{GENERATING ALL SIMPLE CYCLES}

We outline how to generate all simple cycles within an SCC. Let $T$ be the next-transition-tree of an arbitrary node in this SCC.

We process each root-to-leaf path of $T$ as follows: if this path contains (exactly) two instances of the same node then the path segment connecting these two instances corresponds to a simple cycle. It can be shown that this procedure gives all possible cycles. (Proof Sketch: let $C=v_{1} v_{2} v_{3} \cdots v_{r} v_{1}$ be any simple cycle. Find a node $v_{i}$ of $C$ that is closest to the root in $T$. If there are multiple nodes all closest to the root, pick any one of them. $v_{i}$ must contain an edge to $v_{i+2}$, which in turn must contain an edge to $v_{i+1}$ and so on. Inductively, one can show that the subtree of $T$ rooted at $v_{i}$ contains the path $v_{i} v_{i+1} \cdots v_{r} v_{1} v_{2} v_{i-1}$.) However, the same cycle may appear more than once. So we need to maintain a set data structure of all cycles generated. Before inserting a new cycle, we check whether it is already present or not.

\section{GENERATING ALL ACYCLIC PATHS}

As mentioned earlier, we first find all the strongly connected components (SCC) of the transition graph. Then we construct a component graph where each node represents an SCC and an edge from node $u$ to node $v$ represents an edge (in the original transition graph) from a node belonging to $u$ 's SCC to a node belonging to $v$ 's SCC. This component graph is a Directed Acyclic Graph (DAG), i.e., a graph without any cycles [3]. 
We first generate all acyclic paths in the DAG and then replace each SCC on any given path with a set of acyclic paths within the SCC.

Redundancy. There are a finite number of distinct paths (tests) in a DAG. However, we do not want to test all of them; Our goal is to generate a minimal number of tests, which satisfies the coverage criterion and does not contain any redundant tests.

The tests need to start from the start state, so we only consider paths starting from the source node (node without any incoming transitions). In addition, the following is a very popular redundancy criterion in practice.

(R1) Proper prefixes are redundant and can be discarded.

From the redundancy criterion above, we only consider paths that terminate at a sink node (node without any outgoing transitions). Any path not terminating at a sink node will be a proper prefix of a path that has been extended to a sink node. Combining the above two, we only need to generate paths that start from the source node and terminate at one of the sink nodes.

Figure 3 gives an algorithm for generating all source to sink paths in a DAG.

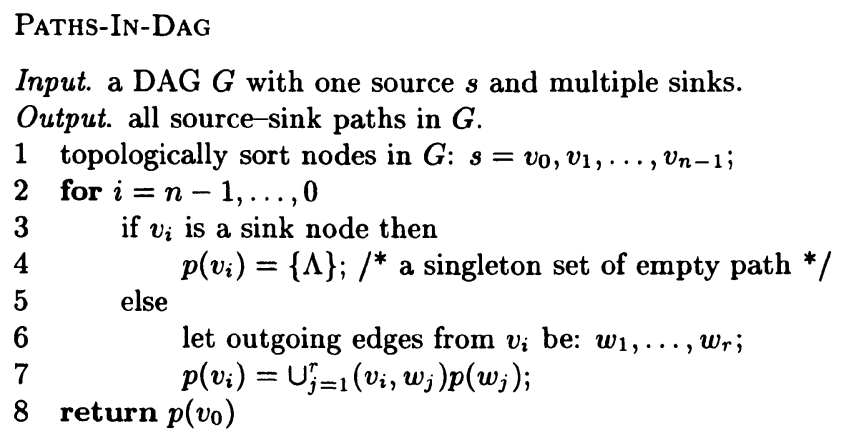

Figure 3 Generating all source-sink paths in a DAG

Generating All Acyclic Paths in a DAG. The algorithm (Figure 3 ) is bottom-up, starting from one of the the sink nodes $t=v_{n-1}$. When processing a node $v_{i}$, we examine all its outgoing edges $\left(v_{i}, w_{j}\right)$ where the paths from $w_{j}$ to sink nodes have been computed. At Line 7 , we concatenate edge $\left(v_{i}, w_{j}\right)$ to each path computed at $w_{j}$ and collect it at node $v_{i}$. After processing the source node $s=v_{0}$, we have obtained all the paths, which are non-redundant and have a complete coverage. 
A topological sort takes time proportional to the number of edges. We account for the examination and concatenation of each edge, which is processed only once at Line 7 , and the total cost is proportional to the total path lengths:

Proposition 1 The algorithm in Figure 3 constructs all source-sink paths. Its time and space requirement is linear in the output (the total lengths of all the constructed tests).

Proposition 2 For machines with a reset to source s, the constructed test sequences are a checking sequence.

Generating All Acyclic Paths within an SCC. Up to now we have discussed test (path) generation for DAGs where nodes can be SCC. For each edge connecting two SCC's on the path, we need to replace it with an edge in the original graph. In general, it will be possible to replace the edge between SCC's with one of the several possible edges in the original graph, and we will obtain a separate path for each choice of the replacement edge. We also need to replace each SCC node with a set of acyclic paths within this SCC. Suppose the incoming and outgoing edges of this SCC node are on nodes $u$ and $v$ (respectively) of this SCC. Then we need to replace this SCC node with all possible acyclic paths from $u$ to $v$ in this SCC. These paths can be generated from nexttransition-tree $(u)$.

Figure 4 summarizes the steps in generating all acyclic paths and Figure 5 gives the algorithm for generating all possible tests.

Remark: Here we are replacing any SCC with all possible acyclic paths. This provides exhaustive coverage but generates lots of tests. In certain applications, we may not need to cover the SCC as thoroughly. There are two other options commonly used in practice.

Chinese Postman Tour. For an SCC, we want to test each edge (transition) at least once and we want to minimize the test sequence length. Such a path is called Chinese Postman Tour [2].

Checking Sequence. A more thorough coverage is provided by a checking sequence, which guarantees the structural isomorphism of the implementation and specification machines [10]. The length of the test sequence is longer than that of a Chinese Postman Tour.

\subsection{REDUNDANCY CRITERIA}

The algorithm outlined in the previous sections (Figure 4) provides exhaustive coverage. However, the number of tests generated is enormous even for systems of moderate size. 


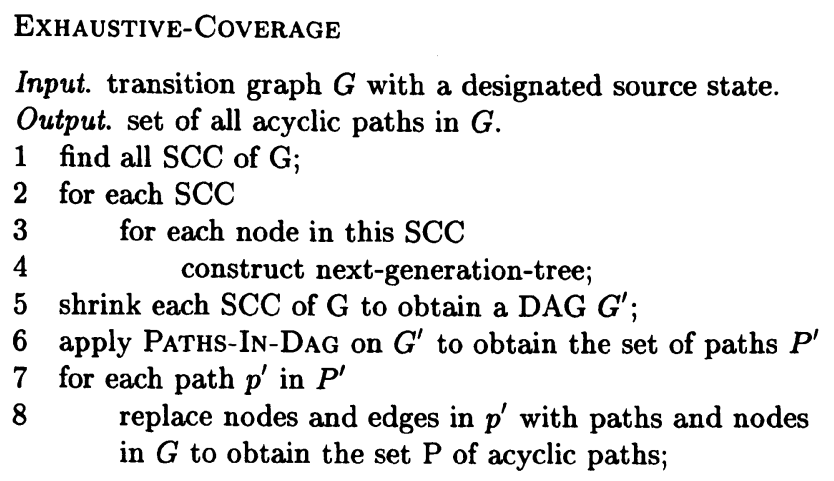

Figure 4 Generating all acyclic paths for exhaustive coverage

\section{EXHAUSTIVE-TEST-GenERATION}

Input. An EFSM of the system.

Output. minimal set of tests providing exhaustive coverage.

1 construct an equivalent FSM and its transition diagram G.

2 apply Exhaustive-Coverage on $G$ to obtain acyclic paths P.

3 obtain the set $\mathrm{C}$ of all simple cycles.

4 combine the sets $P$ and $C$ (as described in Section 2.1).

Figure 5 Generating all tests for exhaustive coverage

As stated in the introduction, a key insight is that that interoperability errors will be introduced only when the gateways are actually talking to each other about a call. We label the transitions as either "white" (local activity) or "black" (involves both gateways).

Thus for interoperability test generation, we do not need to cover white transitions. This motivates additional redundancy criteria.

(R2) Remove all-white test sequences.

(R3) Let $(u, v)$ be the first black edge in the test sequence. Then replace the path from the source node to node $u$ with the shortest path between the source node and node $u$.

(R4) Let $(u, v)$ be the last black edge in the test sequence. Then replace the path from node $v$ to the sink node with the shortest path between node $v$ and the sink node.

(R5) If there is a sequence of white edges, in which each party separately reaches the idle state, terminate the sequence at the last black edge and then use a shortest path to the state "IdleA,IdleB" at the end of the sequence. 
The first three criteria reflect our belief that white transitions are not relevant for interoperability testing. Their only use is to connect (relevant) black transitions. For example, $R 3$ states that the sequence of transitions before the first black transition is not relevant to us, so we might as well replace that part with the shortest all-white sequence. Redundancy criterion $R 4$ is dual of $R 3$. The last criterion recognizes that if both states reach the "idle" state through a sequence of nonrelevant transitions then whatever happens in the rest of the sequence will be tested at the beginning of another generated sequence.

Generating Test Sequences according to $R 1-R 5$. We give an algorithm (Figure 6) for generating all acyclic paths satisfying these criteria.

The algorithm starts with a graph $G$ and generates another graph $G^{\prime}$ such that the set of source-sink paths in $G^{\prime}$ is the same as the set of source-sink paths in $G$ with criteria $R 2-R 5$.

The redundancy criterion $R 3$ says that the path before the first black transition should be replaced with the shortest all-white path.

Lines 1-4 considers all possible black transitions that can be the first in any sequence and adds a marker 'super-edge' for the shortest all-white path from the source node. Line 9 makes sure that a black transition follows a super-edge. The marker 'super-edge' gets replaced on line 10 by the shortest all-white path. This takes care of redundancy criterion R3.

Lines 5-8 perform an analogous function for sink nodes and the last black transitions in the sequence, taking care of redundancy criterion $R 4$.

Criterion $R 2$ is satisfied because line 9 makes sure that there is at least one black transition.

Instead of generating all paths and then processing them according to criterion $R 5$ (as suggested on line 11) the actual implementation makes sure that the incremental construction of paths in procedure EXHAUSTIVE-COVERAGE never generates a path in violation of $R 5$.

Proposition 3 The algorithm in Figure 6 constructs all acyclic paths according to criteria $R 1-R 5$.

\subsection{ADDITIONAL REDUNDANCY CRITERIA}

On most practical systems, we expect algorithm ADEQUATE-Coverage (Figure 6) to generates a considerably smaller set of test sequences than 


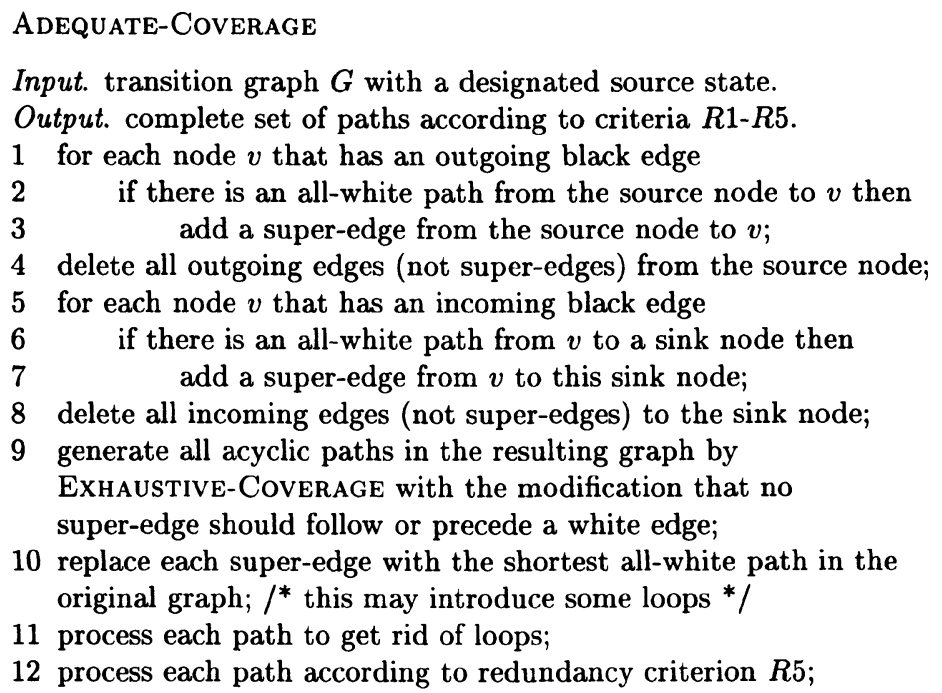

Figure 6 Generating paths for adequate coverage (according to $R 1-R 5$ )

the naive algorithm (Figure 4). Unfortunately even such a smaller set may be too large for manual test execution.

In this section, we suggest a set of more restrictive criteria for test generation that concentrates only on black transitions.

(R6) Generate acyclic paths that consist only of black edges, except that the prefix from the source node and the suffix to the sink node is allowed to contain white edges.

(R7) Generate simple cycles consisting only of black edges.

Generating Test Sequences according to R6-R7. We give an algorithm BASIC-COVERAGE (Figure 7) for generating the test-set according to $R 6$ and $R 7$. The algorithm starts with a graph $G$ and transforms into graph $G^{\prime}$ such that the set of source-sink paths in $G^{\prime}$ is the same as the set of source-sink paths in $G$ with criterion $R 6$. (We do not discuss criterion $R 7$ but it is easy to modify the procedure to generate all simple cycles to satisfy $R 7$.)

The intuition of the algorithm is to delete all white edges except those needed to reach black transitions from the source node (or from the black transitions to the sink nodes). Lines 1-3 maintains a marker 'super-edge' for each source node to black transition path. On line 10, we replace this marker with the shortest path.

Lines 4-6 perform an analogous function for sink nodes. 


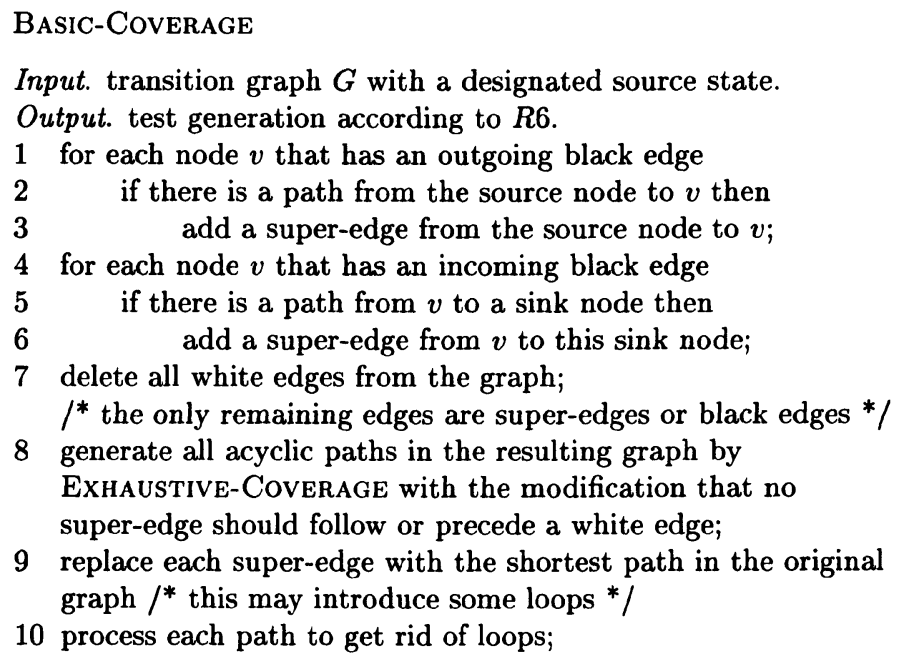

Figure 7 Generating all acyclic paths for basic coverage(according to $R 6$ )

Proposition 4 The algorithm in Figure 7 constructs all acyclic paths according to criterion $R 6$.

Discussion: We feel that both algorithms BASIC-COVERAGE and ADEQUATE-COVERAGE are interesting in their own rights. If automated test execution is available then ADEQUATE-COVERAGE is the preferred algorithm. If on the other hand, test cases have to be executed by hand then BASIC-COVERAGE is likely to produce a manageable set of test sequences. Alternatively, one can always start with BASIC-COVERAGE since it captures the most critical interoperability behavior. If the system passes tests generated by BASIC-COVERAGE, one can move to a broader coverage provided through ADEQUATE-COVERAGE.

\section{ITIS: AN INTEROPERABILITY TEST GENERATION SOFTWARE TOOL}

The algorithms described in earlier sections are part of the ITIS (Interoperability Testing Intelligent System) project at Lucent Bell Laboratories. ITIS is a software tool for automated interoperability test generation. It is inexpensive and completely portable. ITIS is written in ANSI $\mathrm{C}$ and $\mathrm{Tcl} / \mathrm{Tk}$. We first describe its architecture and then report experimental results. 


\subsection{ARCHITECTURE OF ITIS}

ITIS has a GUI for user input and for displaying test sequences. The workflow of ITIS is shown in Figure 8.

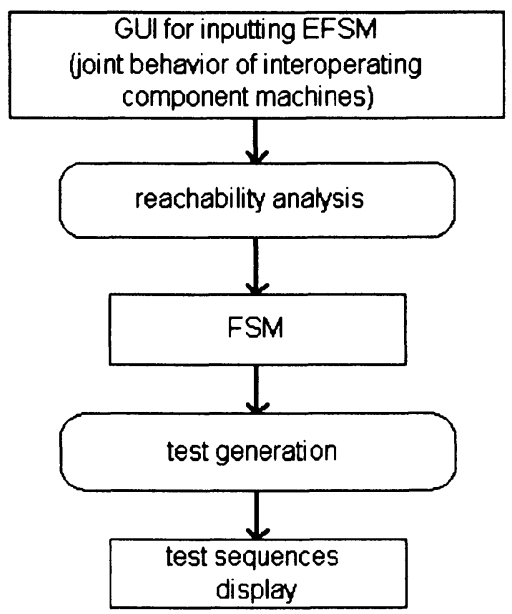

Figure 8 The workflow ITIS for interoperability test generation

The input to ITIS is an Extended Finite State Machine description of the system behavior, of which we have complete information. ITIS will first do reachability analysis to convert the EFSM into an FSM, and then use different algorithms discussed in the above sections to generate test sequences.

\subsection{EXPERIMENTS}

We have used ITIS to generate the interoperability test cases for Enduser VoIP testing.

The EFSM for this protocol has 21 states and 68 transitions, among them 24 transitions are colored as black, others white. The shrunk DAG generated by ITIS from this EFSM contains three SCC nodes; only one of them contains more than one states. The number of generated test sequence by applying different algorithms is shown in the following table. (A longer version of this paer, that includes detailed description of EFSM and the experiments, is available as a Bell labs TM.)

The coverage of the 22 test sequences generated by the algorithm BASIC-COVERAGE is complete with respect to the requirements for the system modeled by the EFSM. The requirements appear in Bellcore's LATA Switching Systems Generic Requirements [1], which includes hundreds of requirements on the behavior of a telephone switching system. 


\begin{tabular}{l|l|l|l}
\hline Algorithms & loop-free paths & loops & $\begin{array}{l}\text { final } \\
\text { tests }\end{array}$ \\
\hline EXHAUSTIVE-COVERAGE & 950 & 424 & 1752 \\
\hline ADEQUATE-COVERAGE & 508 & 424 & 908 \\
\hline BASIC-COVERAGE & 16 & 4 & 22 \\
\hline
\end{tabular}

These include many, such as trunking requirements and power requirements, that are not relevant to Internet telephony and are not modeled by the EFSM. However, there are 40 requirements that are applicable to Internet telephony. All of these are tested by the 22 test sequences generated by BASIC-COVERAGE.

\section{CONCLUSION}

Heterogeneity is one of the prominent features of networking systems, and interoperability is ubiquitous and has become a major hurdle for system reliability and quality of service. Interoperability testing is indispensable for the integration of reactive systems. Conventional conformance testing techniques do not apply since we want to test the system interfaces but not to check implementations vs. specifications.

We propose several algorithms for automatic generation of interoperability testing cases, incorporating a range of coverage and redundancy criteria. The tests generated are complete and non-redundant. They are applied to VoIP interoperability testing, and provide promising results.

\section{References}

[1] LATA switching systems generic requirements: Call processing. Technical Report GR-505-CORE, Bellcore, December 1997.

[2] A. V. Aho, A. T. Dahbura, D. Lee, and M. U. Uyar. An optimization technique for protocol conformance test generation based on UIO sequences and rural chinese postman tours. IEEE Trans. on Communication, 39(11):1604-15, 1991.

[3] A.V. Aho, J. E. Hopcroft, and J. D. Ullman. The Design and Analysis of Computer Algorithms. Addison-Wesley, 1974.

[4] G. Bonnes. IBM OSI interoperability verification services. In IFIP TC6 WG6.1 The 3rd International Workshop on Protocol Test System, 1990.

[5] R. Castanet and O. Kone. Deriving coordinated testers for interoperability. In O. Rafiq, editor, Protocol Test Systems, VI(C-19), 
pages 331-345. Elsevier Science Publisher B. V.(North-Holland), 1994.

[6] J. Gadre, Rohre C, C. Summers, and S. Symington. A COS study of OSI interoperability. Computer Standards and Interfaces, 9(3):217237, 1990.

[7] R. Hao. Protocol Conformance and Interoperability Testing based on Formal Methods. PhD thesis, Tsinghua University, P.R.China, 1997.

[8] S. Kang and M. Kim. Test sequence generation for adaptive interoperability testing. In Proceeding of Protocol Test Systems, VIII, pages 187-200, 1995.

[9] S. Kang and M. Kim. Interoperability test suite derivation for symmetric communication protocols. In Proceeding of FORTE/PSTV'97, 1997.

[10] D. Lee and M. Yannakakis. Principles and methods of testing finite state machines - a survey. The Proceedings of IEEE, 84(8):10891123, August 1996.

[11] G. Luo, G. Bochmann, and A. Petrenko. Test selection based on communicating nondeterministic finite-state machines using a generalized wp-method. IEEE Transactions on S.E., 20(2):149-162, 1994.

[12] N.Arakawa and T. Soneoka. A test case generation method for concurrent programs. In R.J. Heijink J. Kroon and E. Brinksma, editors, Protocol Test Systems, IV, pages 95-106. Elsevier Science Publisher B. V.(North-Holland), 1992.

[13] O Rafiq and R. Castanet. From conformance testing to interoperability testing. In The 3rd International Workshop on Protocol Test Systems, 1990.

[14] G. S. Vermeer and H. Blik. Interoperability testing: Basis for the acceptance of communicating systems. In Protocol Test Systems, VI(C-19). Elsevier Science Publisher B. V.(North-Holland), 1994. 